\title{
Correction to: Robot-assisted laparoscopic pyeloplasty: a single-centre experience
}

\author{
Thomas Charles Wood ${ }^{1} \cdot$ Nicholas Raison $^{2}$ (D) - Oussama Elhage ${ }^{2} \cdot$ Kamran Ahmed $^{2} \cdot$ Declan Cahill $^{3}$. \\ Benjamin J. Challacombe ${ }^{4}$. Muhammad Shamim Khan ${ }^{5}$. Prokar Dasgupta ${ }^{2}$
}

Published online: 25 May 2018

○) Springer Science+Business Media, LLC, part of Springer Nature 2018

\section{Correction to: Surgical Endoscopy}

$$
\text { https://doi.org/10.1007/s00464-018-6212-2 }
$$

In the original version of this article, Oussama Elhage's name was spelled incorrectly. It is correct as displayed above.

The authors regret the error.

The original article can be found online at https://doi.org/10.1007/ s00464-018-6212-2.

Nicholas Raison

nicholas.raison@kcl.ac.uk

Thomas Charles Wood

bsms3330@uni.bsms.ac.uk

Oussama Elhage

oussama.elhage@kcl.ac.uk

Kamran Ahmed

kamran.ahmed@kcl.ac.uk

Declan Cahill

dcahillurology@gmail.com

Benjamin J. Challacombe

benchallacombe@doctors.org.uk

Muhammad Shamim Khan

shamim.khan@gstt.nhs.uk
Prokar Dasgupta

prokarurol@gmail.com

1 Brighton and Sussex Medical School, Audrey Emerton Building, Eastern Road, Brighton BN2 5BE, UK

2 MRC Centre for Transplantation, King's College London, Guy's Hospital, London SE1 9RT, UK

3 Royal Marsden Hospital, London SW3 6JJ, UK

4 Department of Urology, Guy's and St. Thomas' Hospital NHS Foundation Trust, London SE1 9RT, UK

5 Guy's Hospital and King's College, London School of Medicine, London, UK 